\title{
Anomalous fundamental diagrams in traffic on ant trails
}

\author{
Andreas Schadschneider ${ }^{1}$, Debashish Chowdhury ${ }^{2}$, Alexander John ${ }^{1}$, and \\ Katsuhiro Nishinari ${ }^{3}$ \\ 1 Institut für Theoretische Physik, Universität zu Köln, 50937 Köln, Germany, \\ [as , aj] @thp.uni-koeln.de \\ 2 Department of Physics, Indian Institute of Technology, Kanpur 208016, India, \\ debch@iitk.ac.in \\ 3 Department of Applied Mathematics and Informatics, Ryukoku University, Shiga \\ 520-2194, Japan, knishi@rins.ryukoku.ac.jp
}

\begin{abstract}
Many insects like ants communicate chemically via chemotaxis. This allows them to build large trail systems which in many respects are similar to human-build highway networks. Using a recently proposed stochastic cellular automaton model we discuss the basic properties of the traffic flow on existing trails. Surprisingly it is found that in certain regimes the average speed of the ants can vary non-monotonically with their density. This is in sharp contrast to highway traffic. The observations can be understood by the formation of loose clusters, i.e. space regions of enhanced, but not maximal, density. We also discuss the effect of counterflow on the trails.
\end{abstract}

\section{Introduction}

The occurance of organized traffic $[1-3]$ is not only restricted to human societies. Prominent examples of self-organized motion in biology are herding, flocking and swarm formation [4-7]. In addition, especially ants build trail systems that have many similarities with highway networks [8].

Ants communicate with each other by dropping a chemical (generically called pheromone) on the substrate as they move forward [9-11]. Although we cannot smell it, the trail pheromone sticks to the substrate long enough for the other following sniffing ants to pick up its smell and follow the trail. This process is called chemotaxis [7]. Ant trails may serve different purposes (trunk trails, migratory routes) and may also be used in a different way by different species. Therefore one-way trails are observed as well as trails with counterflow of ants.

In the following we will not discuss the process of trail formation itself. This has been studied in the past (see e.g. $[12,13]$ and references therein). Instead we assume the existence of a trail network that is constantly reinforced by the ants. Focussing on one particular trail it is natural to assume that the motion of the ants is one-dimensional.

In traffic flow interactions between the vehicles typically lead to a reduction of the average velocity $\bar{v}$. This is obvious for braking maneouvers to avoid crashes. Therefore $\bar{v}(\rho)$ decreases with increasing density $\rho .{ }^{1}$ In the following we will show

\footnotetext{
${ }^{1}$ A possible exception is a synchronized phase where the correlation between current and density is very small [14].
} 
that this can be different for ant-trails. Even though due to the similar velocity of the ants overtaking is very rare, which implies that a description in terms of an exclusion process is possible, the presence of the pheromone effectively leads to an enhancement of the velocity.

We just briefly mention that similar ideas can be applied to the description of pedestrian dynamics. In $[15,16]$ we have developed a pedestrian model based on virtual chemotaxis. The basic principles are very similar to the ant-trail model introduced below. However, the motion in pedestrian dynamics is essentially two-dimensional.

\section{Definition of the model}

In [17-19] we have developed a particle-hopping model, formulated in terms of a stochastic cellular automaton (CA), which may be interpreted as a model of uni-directional flow in an ant-trail. As mentioned in Sec. 1 we do not want to address the question of the emergence of the ant-trail [20], but focus on the traffic of ants on a trail which has already been formed. The model generalizes the asymmetric simple exclusion process (ASEP) [21-23] with parallel dynamics by taking into account the effect of the pheromone.

The ASEP is one of the simplest examples of a system driven far from equilibrium. Space is discretized into cells that can be occupied by at most one particle. In the totally asymmetric case (TASEP) particles are allowed to move in one direction only, e.g. to the right. If the right neighbour site is empty a particle hops there with probability $q$. For parallel (synchronous) dynamics this is identical to the limit $v_{\max }=1$ of the Nagel-Schreckenberg (NaSch) model [24] with braking probability $p=1-q$.

In our model of uni-directional ant-traffic the ants move according to a rule which is essentially an extension of the TASEP dynamics. In addition a second field is introduced which models the presence or absence of pheromones (see Fig. 1). The hopping probability of the ants is now modified by the presence of pheromones. It is larger if a pheromone is present at the destination site. Furthermore the dynamics of the pheromones has to be specified. They are created by ants and free pheromones evaporate with probability $f$. Assuming periodic boundary conditions, the state of the system is updated at each time step in two stages (see Fig. 1). In stage I ants are allowed to move while in stage II the pheromones are allowed to evaporate. In each stage the stochastic dynamical rules are applied in parallel to all ants and pheromones, respectively.

\section{Stage I: Motion of ants}

An ant in a cell cannot move if the cell immediately in front of it is also occupied by another ant. However, when this cell is not occupied by any other ant, the probability of its forward movement to the ant-free cell is $Q$ or $q$, depending on whether or not the target cell contains pheromone. Thus, $q$ (or $Q$ ) would be the average speed of a free ant in the absence (or presence) of pheromone. To be consistent with real ant-trails, we assume $q<Q$, as presence of pheromone 

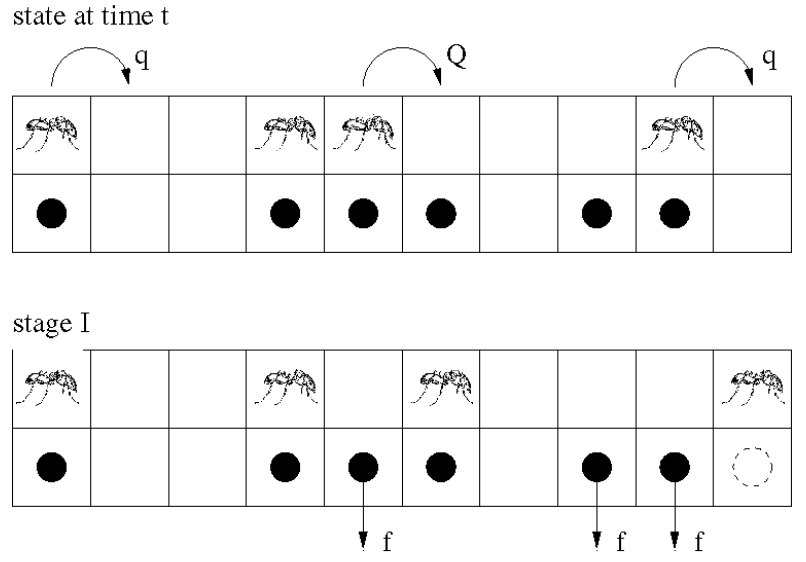

stage II

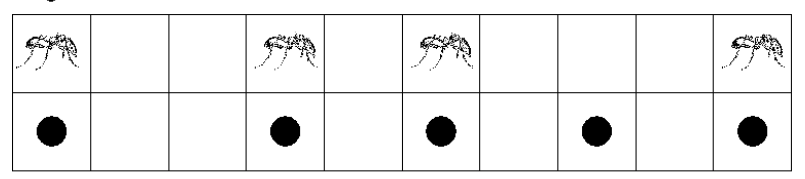

state at time $t+1$

Fig. 1. Schematic representation of typical configurations of the uni-directional anttraffic model. The symbols • indicate the presence of pheromone. This figure also illustrates the update procedure. Top: Configuration at time $t$, i.e. before stage $I$ of the update. The non-vanishing probabilities of forward movement of the ants are also shown explicitly. Middle: Configuration after one possible realisation of stage I. Two ants have moved compared to the top part of the figure. The open circle with dashed boundary indicates the location where pheromone will be dropped by the corresponding ant at stage $I I$ of the update scheme. Also indicated are the existing pheromones that may evaporate in stage $I I$ of the updating, together with the average rate of evaporation. Bottom: Configuration after one possible realization of stage II. Two drops of pheromones have evaporated and pheromones have been dropped/reinforced at the current locations of the ants.

increases the average speed.

\section{Stage II: Evaporation of pheromones}

Trail pheromone is volatile. So, pheromone secreted by an ant will gradually decay unless reinforced by the following ants. In order to capture this process, we assume that each cell occupied by an ant at the end of stage I also contains pheromone. On the other hand, pheromone in any 'ant-free' cell is allowed to evaporate; this evaporation is also assumed to be a random process that takes place at an average rate of $f$ per unit time.

The total amount of pheromone on the trail can fluctuate although the total number $N$ of the ants is constant because of the periodic boundary conditions. In 
the two special cases $f=0$ and $f=1$ the stationary state of the model becomes identical to that of the TASEP with hopping probability $Q$ and $q$, respectively.

For a theoretical description of the process we associate two binary variables $S_{i}$ and $\sigma_{i}$ with each site $i . S_{i}$ is the occupation number of ants and takes the value 0 or 1 depending on whether the cell $i$ is empty or occupied by an ant. Similarly, $\sigma_{i}$ is the occupation number of the pheromones, i.e. $\sigma_{i}=1$ if the cell $i$ contains pheromone; otherwise, $\sigma_{i}=0$. Thus, we have two subsets of dynamical variables in this model, namely, $\{S(t)\} \equiv\left\{S_{1}(t), S_{2}(t), \ldots, S_{i}(t), \ldots, S_{L}(t)\right\}$ and $\{\sigma(t)\} \equiv\left\{\sigma_{1}(t), \sigma_{2}(t), \ldots, \sigma_{i}(t), \ldots, \sigma_{L}(t)\right\}$. In stage I the subset $\{S(t+1)\}$ at the time step $t+1$ is obtained using the full information $(\{S(t)\},\{\sigma(t)\})$ at time $t$. In stage II only the subset $\{\sigma(t)\}$ is updated so that at the end of stage II the new configuration $(\{S(t+1)\},\{\sigma(t+1)\})$ at time $t+1$ is obtained.

The rules can be written in a compact form as the coupled equations

$$
\begin{gathered}
S_{j}(t+1)=S_{j}(t)+\min \left\{\eta_{j-1}(t), S_{j-1}(t), 1-S_{j}(t)\right\} \\
-\min \left\{\eta_{j}(t), S_{j}(t), 1-S_{j+1}(t)\right\}, \\
\sigma_{j}(t+1)=\max \left\{S_{j}(t+1), \min \left\{\sigma_{j}(t), \xi_{j}(t)\right\}\right\},
\end{gathered}
$$

where $\xi$ and $\eta$ are stochastic variables defined by $\xi_{j}(t)=0$ with the probability $f$ and $\xi_{j}(t)=1$ with $1-f$, and $\eta_{j}(t)=1$ with the probability $p=q+(Q-q) \sigma_{j+1}(t)$ and $\eta_{j}(t)=0$ with $1-p$. This representation is useful for the development of approximation schemes.

\section{Fundamental diagram for uni-directional motion}

In vehicular traffic, usually, the inter-vehicle interactions tend to hinder each other's motion so that the average speed of the vehicles decreases monotonically with increasing density. This can be seen in Fig. 2 where also the fundamental diagram of the NaSch model with $v_{\max }=1$ (or the TASEP with parallel updating) is shown for different hopping probabilities. Note also the particle-hole symmetry of the flow that satisfies $F_{\mathrm{NS}}(\rho)=F_{\mathrm{NS}}(1-\rho)$. Explicitly it is given by [25]

$$
F_{\mathrm{NS}}(\rho)=\frac{1}{2}\left[1-\sqrt{1-4 q_{\mathrm{NS}} \rho(1-\rho)}\right]
$$

where $q_{\mathrm{NS}}=1-p$ with $p$ being the braking probability. Here $\rho=N / L$ is the density of vehicles and $L$ the number of cells.

In contrast, in our model of uni-directional ant-traffic the average speed of the ants varies non-monotonically with their density over a wide range of small values of $f$ (see Fig. 2) because of the coupling of their dynamics with that of the pheromone. This uncommon variation of the average speed gives rise to the unusual dependence of the flux on the density of the ants in our unidirectional ant-traffic model (Fig. 2). Furthermore the flux is no longer particlehole symmetric. 

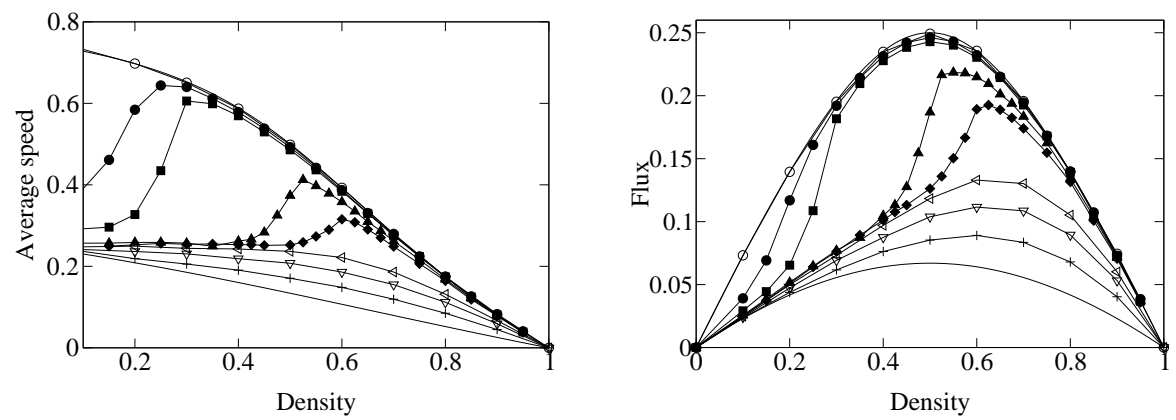

Fig. 2. The average speed (left) and the flux (right) of the ants, in the uni-directional ant-traffic model, are plotted against their densities for the parameters $Q=0.75, q=$ 0.25. The curves correspond to $f=0.0001(\circ), 0.0005(\bullet), 0.001(\boldsymbol{\square}), 0.005(\boldsymbol{\Delta}) 0.01(\bullet)$, $0.05(\triangleleft), 0.10(\nabla), 0.25(+)$. In both graphs, the cases $f=0$ and $f=1$ are also displayed by the uppermost and lowermost curves (without points); these are exact results corresponding to the TASEP or NaSch model with $v_{\max }=1$ and hopping probability $Q$ and $q$, respectively. Curves plotted with filled symbols have unusual shapes.
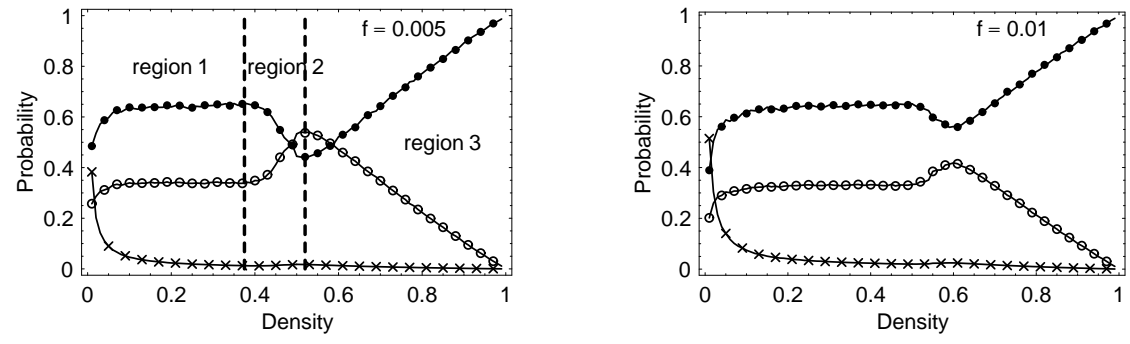

Fig. 3. Numerical results for the probabilities of finding an ant $(\bullet)$, pheromone but no ant $(\circ)$ and nothing $(\times)$ in front of an ant are plotted against density of the ants. The parameters are $f=0.005$ (left) and $f=0.01$ (right).

\section{1 "Loose" cluster approximation (LCA)}

How can the unusual density-dependence of the average velocity be understood? Using mean-field-type theories that implicitly assume a homogeneous stationary state does not allow to reproduce the simulation results in a satisfactory way [18]. This indicates that the stationary state is characterized by some sort of clustering. This is confirmed by considering the probabilities of finding an ant $\left(P_{a}\right)$, pheromone $\left(P_{p}\right)$ and nothing $\left(P_{0}\right)$ in front of a cell occupied by an ant. Typical results from computer simulations are shown in Fig. 3.

One can distinguish three different regions. "Region 1" is characterized by the flat part of the curves in Fig. 3 in the low density regime. Here, in spite of low density of the ants, the probability of finding an ant in front of another is quite high. This implies the fact that ants tend to form a cluster. On the other 


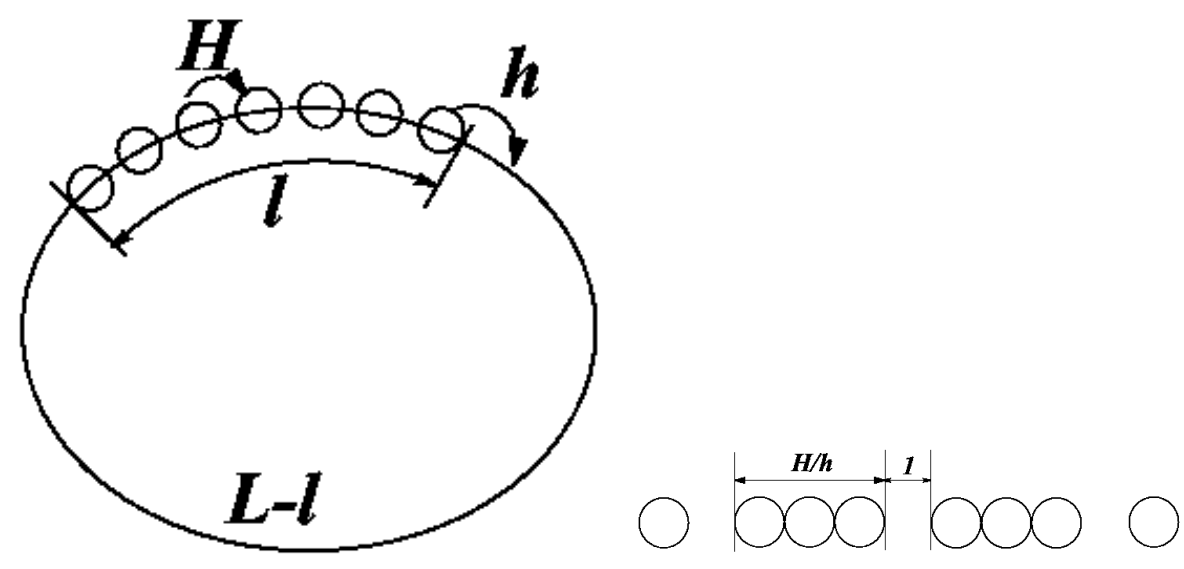

Fig. 4. (left) Schematic explanation of the loose cluster. $H$ is the hopping probability of ants inside the loose cluster and $h$ is that of the leading ant. (right) The stationary loose cluster. The average gap between ants becomes $h / H$, which is irrelevant to the density of ants.

hand, cluster-size distributions obtained from computer simulations show that the probability of finding isolated ants are always higher than that of finding a cluster of ants occupying nearest-neighbor sites [18]. These two apparently contradictory observations can be reconciled by assuming that the ants form "loose" clusters in the region 1 . The term "loose" means that there are small gaps in between successive ants in the cluster, and the cluster looks like an usual compact cluster if it is seen from a distance (Fig. 4). In other words, a loose cluster is just a loose assembly of isolated ants that corresponds to a space region with density larger than the average density $\rho$, but smaller than the maximal density $(\rho=1)$ of a compact cluster.

Let us assume that the loose cluster becomes stationary after sufficient time has passed. Then the hopping probability of all the ants, except the leading one, is assumed to be $H$, while that of the leading one is $h$ (see Fig. 4). The values of $H$ and $h$ have to be determined self-consistently. If $f$ is small enough, then $H$ will be close to $Q$ because the gap between ants is quite small. On the other hand, if the density of ants is low enough, then $h$ will be very close to $q$ because the pheromone dropped by the leading ant would evaporate when the following ant arrives there.

The typical size of the gap between successive ants in the cluster can be estimated [18] by considering a simple time evolution beginning with a usual compact cluster (with local density $\rho=1$ ). The leading ant will move forward by one site over the time interval $1 / h$. This hopping occurs repeatedly and in the interval of the successive hopping, the number of the following ants which will move one step is $H / h$. Thus, in the stationary state, strings (compact clusters) of length $H / h$, separated from each other by one vacant site, will produced repeatedly by the ants (see Fig. 4). Then the average gap between ants is $\frac{(H / h-1) \cdot 0+1 \cdot 1}{H / h}=\frac{h}{H}$, in- 
dependent of their density $\rho$. Interestingly, the density-independent average gap in the LCA is consistent with the flat part (i.e., region 1) observed in computer simulations (Fig. 3). In other words, region 1 is dominated by loose clusters.

Beyond region 1, the effect of pheromone of the last ant becomes dominant. Then the hopping probability of leading ants becomes large and the gap becomes wider, which will increase the flow. We call this region as region 2 , in which "looser" clusters are formed in the stationary state. It can be characterized by a negative gradient of the density dependence of the probability to find an ant in front of a cell occupied by an ant (see Fig. 3).

Considering these facts, we obtain the following equations for $h$ and $H$ :

$$
\left(\frac{h-q}{Q-q}\right)^{h}=(1-f)^{L-l}, \quad\left(\frac{H-q}{Q-q}\right)^{H}=(1-f)^{\frac{h}{H}}
$$

where $l$ is the length of the cluster given by $l=\rho L+(\rho L-1) \frac{h}{H}$. These equations can be applied to the region 1 and 2 .

The total flux in this system is then calculated as follows. The effective density $\rho_{\text {eff }}$ in the loose cluster is given by $\rho_{\text {eff }}=\frac{1}{1+h / H}$. Therefore, considering the fact that there are no ants in the part of the length $L-l$, the total flux $F$ is

$$
F=\frac{l}{L} f\left(H, \rho_{\mathrm{eff}}\right),
$$

where $f\left(H, \rho_{\text {eff }}\right)$ is given by

$$
f\left(H, \rho_{\mathrm{eff}}\right)=\frac{1}{2}\left(1-\sqrt{1-4 H \rho_{\mathrm{eff}}\left(1-\rho_{\mathrm{eff}}\right)}\right) .
$$

Above the density $\rho=1 / 2$, ants are assumed to be uniformly distributed such that a mean-field approximation works well [18]. We call this region as region 3. Thus we have three typical regions in this model. In region 3 , the relation $H=h$ holds because all the gaps have the same length, i.e. the state is homogeneous. Thus $h$ is determined by

$$
\left(\frac{h-q}{Q-q}\right)^{h}=(1-f)^{\frac{1}{\rho}-1}
$$

which is the same as our previous paper, and flux is given by $f(h, \rho)$. It is noted that if we put $\rho=1 / 2$ and $H=h$, then (4) coincides with (7).

In region 1 we can simplify the analysis by assuming $h=q$ in (4). Then the flux-density relation becomes linear. Numerical results for regions 1 and 2 can be obtained by solving (4) using the Newton method for densities $\rho \leq 1 / 2$. Above this value of density, equation (7) can be used. Together these approximations can reproduce the results of the simulations rather well [18].

Another interesting phenomenon observed in the simulations is coarsening. At intermediate time usually several loose clusters are formed (Fig. 5). However, the velocity of a cluster depends on the distance to the next cluster ahead. Obviously, the probability that the pheromone created by the last ant of the 

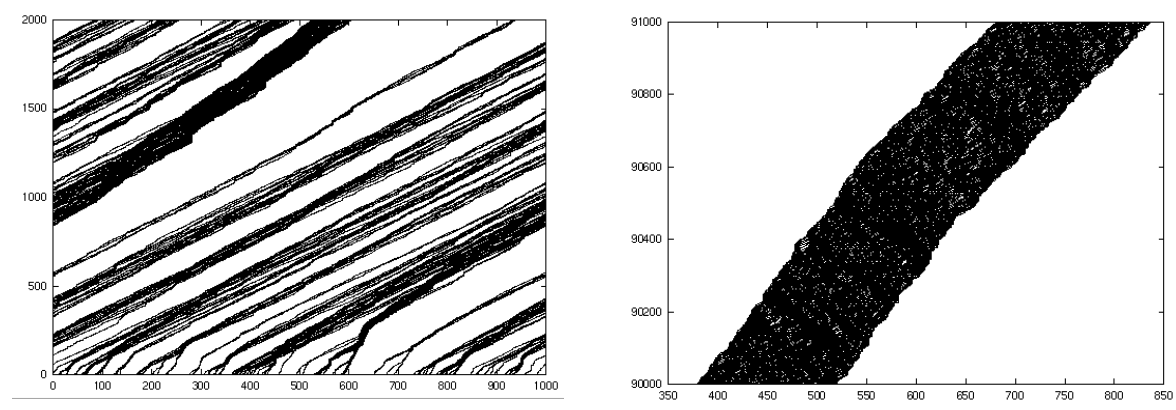

Fig. 5. Space-time plots showing the coarsening process of the loose clusters. The left part shows the state of a system of $L=1000$ cells and $N=100$ ants at early times. The right part corresponds to the stationary state. The evaporation probability of the pheromones is $f=0.0005$.

previous cluster survives decreases with increasing distance. Therefore clusters with a small headway move faster than those with a large headway. This induces a coarsening process such that after long times only one loose cluster survives (Fig. 5). A similar behaviour has been observed in the bus-route model [26,27].

\section{Bidirectional motion}

We develope a model of bi-directional ant-traffic [19] by extending the model of uni-directional ant-traffic described in Sec. 2. In the models of bi-directional anttraffic the trail consists of two lanes of cells (see Fig. 6). These two lanes need not be physically separate rigid lanes in real space; these are, however, convenient for describing the movements of ants in two opposite directions. In the initial configuration, a randomly selected subset of the ants move in the clockwise direction in one lane while the others move counterclockwise in the other lane. However, ants are allowed neither to take U-turn ${ }^{2}$ nor to change lane. Thus, the ratio of the populations of clockwise-moving and anti-clockwise moving ants remains unchanged as the system evolves with time. All the numerical data presented here are for the symmetric case where equal number of ants move in the two directions. Therefore, the average flux of outbound and nestbound ants are identical. In all the graphs we plot only the flux of the nestbound ants.

The rules governing the dropping and evaporation of pheromone in the model of bi-directional ant-traffic are identical to those in the model of uni-directional traffic. The common pheromone trail is created and reinforced by both the outbound and nestbound ants. The probabilities of forward movement of the ants in the model of bi-directional ant-traffic are also natural extensions of the similar situations in the uni-directional traffic. When an ant (in either of the two lanes) does not face any other ant approaching it from the opposite direction the

\footnotetext{
${ }^{2}$ U-turns of so-called followers on pre-existing trails are very rare [28].
} 


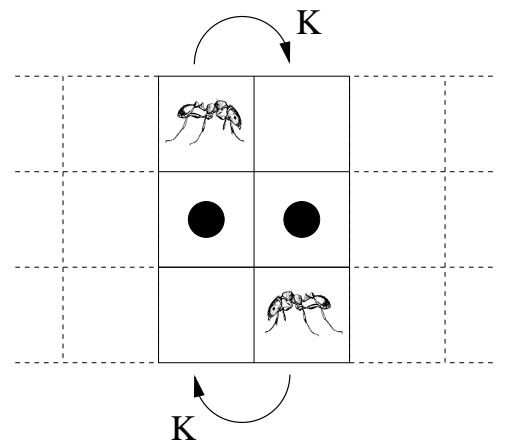

Fig. 6. A typical head-on encounter of two oppositely moving ants in the model of $b i$-directional ant-traffic. This is a totally new process which does not have any analog in the model of uni-directional ant-traffic.

likelihood of its forward movement onto the ant-free cell immediately in front of it is $Q$ or $q$, respectively, depending on whether or not it finds pheromone ahead. Finally, if an ant finds another oncoming ant just in front of it, as shown in Fig. 6, it moves forward onto the next cell with probability $K$.

Since ants do not segregate in perfectly well defined lanes, head-on encounters of oppositely moving individuals occur quite often although the frequency of such encounters and the lane discipline varies from one species of ants to another. In reality, two ants approaching each other feel the hindrance, turn by a small angle to avoid head-on collision [13] and, eventually, pass each other. At first sight, it may appear that the ants in our model follow perfect lane discipline and, hence, unrealistic. However, that is not true. The violation of lane discipline and head-on encounters of oppositely moving ants is captured, effectively, in an indirect manner by assuming $K<Q$. But, a left-moving (right-moving) ant cannot overtake another left-moving (right-moving) ant immediately in front of it in the same lane. It is worth mentioning that even in the limit $K=Q$ the traffic dynamics on the two lanes would remain coupled because the pheromone dropped by the outbound ants also influence the nestbound ants and vice versa.

Fig. 7 shows fundamental diagrams for the two relevant cases $q<K<Q$ and $K<q<Q$ and different values of the evaporation probability $f$. In both cases the unusual behaviour related to a non-monotonic variation of the average speed with density as in the uni-directional model can be observed [19].

An additional feature of the fundamental diagram in the bi-directional anttraffic model is the occurrence of a plateau region. This plateau formation is more pronounced in the case $K<q<Q$ than for $q<K<Q$ since they appear for all values of $f$. Similar plateaus have been observed earlier $[29,30]$ in models related to vehicular traffic where randomly placed bottlenecks slow down the traffic in certain locations along the route. Note that for $q<K<Q$ (Fig. 7(left)) the plateaus appear only in the two limits $f \rightarrow 0$ and $f \rightarrow 1$, but not for an intermediate range of values of $f$. In the limit $f \rightarrow 0$, most often the likelihood of the forward movement of the ants is $Q$ whereas they are forced to 

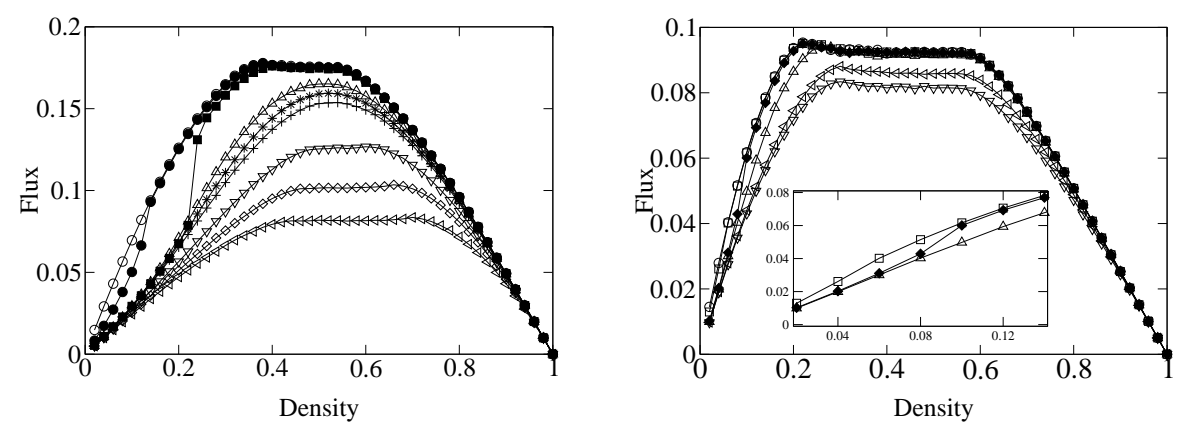

Fig. 7. Fundamental diagrams of the model for bi-directional traffic for the cases $q<$ $K<Q$ (left) and $K<q<Q$ (right) for several different values of the pheromone evaporation probability $f$. The parameters in the left graph are $Q=0.75, q=0.25$ and $K=0.5$. The symbols $\circ, \bullet, \mathbf{\square}, \triangle, *,+, \nabla, \diamond$ and $\triangleleft$ correspond, respectively, to $f=0,0.0005,0.005,0.05,0.075,0.10,0.25,0.5$ and 1 . The parameters in the right graph are $Q=0.75, q=0.50$ and $K=0.25$. The symbols $\circ, \square, \diamond, \triangle, \triangleleft$ and $\nabla$ correspond, respectively, to $f=0,0.0005,0.005,0.05,0.5$ and 1 . The inset in the right graph is a magnified re-plot of the same data, over a narrow range of density, to emphasize the fact that the unusual trend of variation of flux with density in this case is similar to that observed in the case $q<K<Q$ (left). The lines are merely guides to the eye. In all cases curves plotted with filled symbols exhibit non-monotonic behaviour in the speed-density relation.

move with a smaller probability $K$ at those locations where they face another ant immediately in front approaching from the opposite direction (like the situations depicted in Fig. 6). Thus, such encounters of oppositely moving ants have the same effect on ant-traffic as bottlenecks on vehicular traffic.

But why do the plateaus re-appear for $q<K<Q$ also in the limit $f \rightarrow 1$ ? At sufficiently high densities, oppositely moving ants facing each other move with probability $K$ rather than $q$. In this case, locations where the ants have to move with the lower probability $q$ will be, effectively bottlenecks and hence the re-appearance of the plateau. As $f$ approaches unity there will be larger number of such locations and, hence, the wider will be the plateau. This is consistent with our observation in Fig. 7(left).

\section{Conclusions}

We have introduced a stochastic cellular automaton model of an ant trail [17] characterized by two coupled dynamical variables, representing the ants and the pheromone. With periodic boundary conditions, the total number of ants is conserved whereas the total number of pheromones is not conserved. The coupling leads to surprising results, especially an anomalous fundamental diagram. This anomalous shape of the fundamental diagram is a consequence of the non- 
monotonic variation of the average speed of the ants with their density in an intermediate range of the rate of pheromone evaporation.

As the reason for this unusual behaviour we have identified the special spatiotemporal organization of the ants and pheromone in the stationary state. Three different regimes of density can be distinguished by studying appropriate correlation functions. At low densities (region 1) the behaviour is dominated by the existence of loose clusters which are formed through the interplay between the dynamics of ants and pheromone. These loose clusters are regions with a density that is larger than the average density $\rho$, but not maximal. In region 2 , occuring at intermediate densities, the enhancement of the hopping probability due to pheromone is dominant. Finally, in region 3, at large densities the mutual hindrance against the movements of the ants dominates the flow behaviour leading to a homogeneous state similar to that of the NaSch model.

Furthermore we have introduced a model of bi-directional ant-traffic. The two main theoretical predictions of this model are as follows:

(i) The average speed of the ants varies non-monotonically with their density over a wide range of rates of pheromone evaporation. This unusual variation gives rise to the uncommon shape of the flux-versus-density diagrams.

(ii) Over some regions of parameter space, the flux exhibits plateaus when plotted against density.

In principle, it should be possible to test these theoretical predictions experimentally. Interestingly, various aspects of locomotion of individual ants have been studied in quite great detail [31-33]. However, traffic is a collective phenomenon involving a large number of interacting ants. Surprisingly, to our knowledge, the results published by Burd et al. [34] on the leaf-cutting ant Atta Cephalotes are the only set of experimental data available on the fundamental diagram. Unfortunately, the fluctuations in the data are too high to make any direct comparison with our theoretical predictions. Nevertheless it should be possible to observe the predicted non-monotonicity in future experiments. A simple estimate shows that the typical magnitudes of $f$, for which the non-monotonic variation of the average speed with density is predicted, correspond to pheromone lifetimes in the range from few minutes to tens of minutes. This is of the same order of magnitude as the measured lifetimes of real ant pheromones!

\section{Acknowledgments}

The work of DC is supported, in part, by DFG through a joint Indo-German research grant.

\section{References}

1. D. Chowdhury, L. Santen and A. Schadschneider,:Phys. Rep. 329, 199 (2000); A. Schadschneider: Physica A 313, 153 (2002)

2. D. Helbing: Rev. Mod. Phys. 73, 1067 (2001)

3. T. Nagatani: Rep. Prog. Phys. 65, 1331 (2002)

4. J.K. Parrish, L. Edelstein-Keshet: Science 284, 99 (1999) 
5. Y. Tu: Physica 281, 30 (2000)

6. E.M. Rauch, M.M. Millonas, D.R. Chialvo: Phys. Lett. A207, 185 (1995)

7. E. Ben-Jacob: Contemp. Phys. 38, 205 (1997)

8. N.R. Franks: in Insect Movement: Mechanisms and Consequences (Eds.: I.P. Woiwod, D.R. Reynolds, C.D. Thomas), CAB International (2001)

9. E.O. Wilson: The insect societies (Belknap, Cambridge, USA, 1971); B. Hölldobler and E.O. Wilson: The ants (Belknap, Cambridge, USA, 1990)

10. S. Camazine, J.L. Deneubourg, N. R. Franks, J. Sneyd, G. Theraulaz, E. Bonabeau: Self-organization in Biological Systems (Princeton University Press, 2001)

11. A.S. Mikhailov, V. Calenbuhr: From Cells to Societies, (Springer, 2002)

12. F. Schweitzer: Brownian Agents and Active Particles, Springer Series in Synergetics (Springer 2003)

13. I.D. Couzin, N.R. Franks: Proc. Roy. Soc. London B270, 139 (2003)

14. see e.g. B.S. Kerner: Netw. Spatial Econ. 1, 35 (2001) and these proceedings

15. C. Burstedde, K. Klauck, A. Schadschneider, J. Zittartz: Physica A295, 507 (2001)

16. A. Kirchner, K. Nishinari, A. Schadschneider: Phys. Rev. E67, 056122 (2003)

17. D. Chowdhury, V. Guttal, K. Nishinari, A. Schadschneider: J. Phys. A:Math. Gen. 35, L573 (2002)

18. K. Nishinari, D. Chowdhury, A. Schadschneider: Phys. Rev. E67, 036120 (2003)

19. A. John, A. Schadschneider, D. Chowdhury, K. Nishinari: submitted for publication

20. D. Helbing, F. Schweitzer, J. Keltsch, P. Molnar: Phys. Rev. E56, 2527 (1997)

21. B. Derrida: Phys. Rep. 301, 65 (1998)

22. B. Derrida and M.R. Evans: in Nonequilibrium Statistical Mechanics in One Dimension, ed. V. Privman (Cambridge University Press, 1997).

23. G. Schütz: in Phase Transitions and Critical Phenomena, Vol.19, eds. C. Domb and J.L. Lebowitz (Academic Press, 2000)

24. K. Nagel and M. Schreckenberg, J. Phys. I, 2, 2221 (1992)

25. M. Schreckenberg, A. Schadschneider, K. Nagel, N. Ito: Phys. Rev. E 51, 2939 (1995); A. Schadschneider, M. Schreckenberg: J. Phys. A 26, L679 (1993); A. Schadschneider: Eur. Phys. J. B 10, 573 (1999)

26. O.J. O’Loan, M.R. Evans, M.E. Cates: Phys. Rev. E 58, 1404 (1998); Europhys. Lett. 42, 137 (1998)

27. D. Chowdhury, R.C. Desai: Eur. Phys. J. B 15, 375 (2000)

28. R. Beckers, J.L. Deneubourg, S. Goss: J. Theor. Biol. 159, 397 (1992)

29. S.A. Janowski, J.L. Lebowitz: Phys. Rev. A45, 618 (1992); J. Stat. Phys. 77, 35 (1994)

30. G. Tripathy, M. Barma: Phys. Rev. Lett. 78, 3039 (1997)

31. J.R.B. Lighton, G.A. Bartholomew, D.H. Feener: Physiol. Zoology 60, 524 (1987)

32. C.P.E. Zollikofer: J. Experimental Biology 192, 95 (1994)

33. J.A. Weier, D.H. Feener, J.R.B. Lighton: J. Insect Physiology 41, 321 (1995)

34. M. Burd, D. Archer, N. Aranwela, D. J. Stradling: American Natur. 159, 283 (2002) 